\title{
Investigando modelos pedagógicos para educação a distância: desafios e aspectos emergentes
}

\author{
Investigating educational models for distance education: \\ challenges and emerging issues
}
Investigando modelos educativos de educación a distancia: desafios y cuestiones emergentes

\author{
MARIA IVANICE VENDRUSCOLO* \\ PATRÍCIA ALEJANDRA BEHAR**
}

\begin{abstract}
RESUMO
O estudo apresenta uma investigação qualitativa a respeito de modelos pedagógicos com ênfase na educação a distância, a fim de identificar seus aspectos emergentes. Os avanços sociais, econômicos, culturais e tecnológicos vêm provocando mudanças nas práticas pedagógicas. Consequentemente, provocam o surgimento de novos modelos pedagógicos. Para tal, os dados do estudo foram obtidos no Simpósio Brasileiro de Informática na Educação, relativos ao período de 2001 a 2014, por pesquisa bibliográfica. E os resultados evidenciam que, de forma abrangente, os modelos pedagógicos da amostra atendem às características da geração emergente da $\mathrm{EAD}$, com ênfase na aprendizagem centrada no estudante: o primado da flexibilidade, o primado da interação e o princípio da inclusão digital. Contudo, observou-se, adicionalmente, a não consolidação conceitual do termo modelo pedagógico pela comunidade científica, sendo empregado para designar, principalmente, estratégias e ferramentas pedagógicas.

Palavras-chave: Modelo pedagógico. Educação a distância. Formação de professores.
\end{abstract}

\begin{abstract}
This study presents a qualitative research on pedagogical models with an emphasis on distance education in order to identify their emerging aspects. Social, economic, cultural and technological advances have been causing changes in teaching practices. Consequently, cause the emergence of new pedagogical models. Data for the study were obtained from the Brazilian Symposium on Computer Education for the period 2001-2014, for bibliographic research. The results show that in a comprehensive manner, the pedagogical models of the sample meet the characteristics of the emerging generation of distance education, with an emphasis on student-centered learning, the primacy of flexibility, the primacy of interaction and the principle of digital inclusion. However, it has been observed, further, the non conceptual consolidation term teaching model by the scientific community, it is used to designate mainly education strategies and tools.
\end{abstract}

Keywords: Pedagogical model. Distance education. Teacher training.

\section{RESUMEN}

Este estudio presenta una investigación cualitativa sobre modelos pedagógicos con un énfasis en la educación a distancia, con el fin de identificar sus aspectos emergentes. Avances sociales, económicos, culturales y tecnológicos han dado lugar a cambios en las prácticas de enseñanza. En consecuencia, causar la aparición de nuevos modelos pedagógicos. Los datos para el estudio se obtuvieron del Simposio Brasileño de Informática Educativa para el período 2001-2014, para la literatura. Los resultados muestran que de una manera global, los modelos pedagógicos de la muestra cumplen con las características de la generación emergente de la educación a distancia, con un énfasis en el aprendizaje centrado en el estudiante, la primacía de la flexibilidad, la primacía de la interacción y el principio de la inclusión digital. Sin embargo, se ha observado, además, el modelo de enseñanza no conceptual plazo de consolidación por la comunidad científica, y se utiliza para designar principalmente las estrategias y herramientas de educación.

Palabras clave: Modelo pedagógico. Educación a distancia. Formación del profesorado.

\footnotetext{
*Doutora em Informática na Educação, Professora do Programa de Pós Graduação em Controladoria e Contabilidade (PPGCONT) da Universidade Federal do Rio Grande do Sul.E-mail: <maria.ivanice@ufrgs.br>.

**Doutora em Ciências da Computação. Professora da Faculdade de Educação e dos Cursos de Pós Graduação em Educação (PPGEdu) e em Informática na Educação (PPGIE) da Universidade Federal do Rio Grande do Sul.E-mail: < patricia.behar@ufrgs.br>.
} 


\section{INTRODUÇão}

De forma ampla, modelos são ideias que podem ser expressas por meio de símbolos, palavras, imagens ou ainda por formulações matemáticas para representar um fenômeno. Os modelos científicos representam um recorte teórico para expressar a realidade, ao permitirem a comparação, a simulação e a compreensão dos fenômenos, e possuem três características especiais, na abordagem de Galagovsky e Aduriz-Bravo (2001): (i) são construções temporárias e perfectíveis (nenhum modelo científico tem a verdade absoluta para explicar a realidade); (ii) podem não ser compatíveis entre si (alternativos); e (iii) os modelos alternativos não estão sempre incompatíveis aos modelos anteriores. Logo, os modelos se transformam acompanhando a evolução do próprio conhecimento científico e as mudanças de percepção da realidade (GALAGOVSKY; ADURIZ-BRAVO, 2001; OCAÑA, 2009).

$\mathrm{Na}$ educação, os avanços sociais, econômicos, culturais e tecnológicos vêm provocando mudanças nas práticas pedagógicas (BELLONI, 2009; MORAN, 2009; AMANTE, 2011). Esses avanços, principalmente, das Tecnologias da Informação e da Comunicação (TICs), dos fatores econômicos e de infraestrutura, das políticas educacionais e institucionais, têm contribuído para a criação de um novo sistema de educação aberta a distância (BELLONI, 2009; PETERS, 2009). Consequentemente, provocam o surgimento de novos modelos pedagógicos, decorrentes desse contexto, orientados, principalmente, à aprendizagem e não aos conteúdos (BEHAR, 2009; MORAN, 2009; AMANTE, 2011;AVENDAÑO-CASTRO, 2013). Um exemplo desse modelo é defendido pela Universidade Aberta de Portugal (PEREIRA et al., 2007; BELLONI, 2009).

Os novos modelos pedagógicos têm apresentado características de desenvolvimento das competências (conhecimentos, habilidades e atitudes), construção de conhecimentos, respeito ao ritmo individual, desenvolvimento de autonomia, incentivo à interação, à formação de comunidades de aprendizagem e a redes de convivência (PEREIRA et al., 2007; BELLONI, 2009; BEHAR, 2009; LITTO, 2010; AMANTE, 2011; GOMES, 2013).

Por sua vez, observa-se a necessidade de ampliação da discussão nas Instituições de Ensino Superior (IESs) sobre os novos paradigmas que se abrem adiante da tendência trazida pelos meios digitais e de redefinição dos modelos pedagógicos. Para que, assim, possam refletir sobre as novas formas de comunicação, de relacionamento interpessoal, de ampliação do sentido temporal e espacial, com a conectividade ininterrupta, na educação como um todo, sem dicotomia de modalidades (GOMES, 2013; MOREIRA; BARROS; MONTEIRO, 2014).
Com base nas considerações observadas na realidade educacional, estas suscitam investigações dos modelos pedagógicos aplicados na educação formal e continuada de profissionais, principalmente na Educação a Distância (EAD), a fim de identificar os aspectos emergentes nesse contexto. Logo, questiona-se quais são esses aspectos dos modelos pedagógicos aplicados na EAD? Nesse sentido, o objetivo deste estudo é investigar concepção epistemológica, contribuições teóricas, configurações organizacionais, metodológicas e tecnológicas presentes em trabalhos da comunidade acadêmica relativos aos modelos pedagógicos construídos na EAD.

O estudo justifica-se, primeiramente, pela expansão mundial da EAD, decorrente das novas possibilidades advindas das tecnologias na educação, entre outros fatores, a exemplo da popularização e democratização do acesso às TICs e da dispersão geográfica entre os agentes (PEREIRA et al., 2007; BELLONI, 2009; PETERS, 2009; LITTO 2010). Bem como fundamenta-se, conforme já destacado por Nevado et al. (2001), na necessidade de avaliação dos modelos de formação existentes e de novos parâmetros de melhoria da qualidade do processo educacional.

Para tanto, o artigo está organizado em cinco seções, incluindo esta introdução ao tema. A seção 2 aborda o embasamento teórico sobre modelos pedagógicos, as especificidades da EAD e os trabalhos correlatos. Na seção 3 são expostos os procedimentos metodológicos adotados na investigação. A análise dos dados é apontada na seção 4 e, por fim, na seção 5 , são apresentadas as considerações finais do estudo, com indicação para futuras pesquisas.

\section{CARACTERIZANDO OS MOdELOS PEDAGógICOS PARA EDUCAÇÃO A DISTÂNCIA}

Nesta seção, apresentam-se os fundamentos teóricos que caracterizam os modelos pedagógicos aplicados às especificidades da EAD e os trabalhos correlacionados ao estudo.

\subsection{Definindo e caracterizando o modelo pedagógico}

Inicialmente, pode-se caracterizar um modelo pedagógico como um instrumento de investigação de caráter teórico, construído para representar a idealização do processo de ensino e aprendizagem, contemplando os princípios estruturais e regulamentares desse processo (LOAIZA; RAMÍREZ; LINDO, 2001; MORENO, 2003; OCANÃ, 2009; AVENDAÑO-CASTRO, 2013). Para tanto, conforme esses autores, esse modelo deve possibilitar a interpretação, a projeção e o ajustamento da realidade pedagógica, com embasamento científico e 
ideológico, de forma a reunir os fundamentos teóricos orientadores das práticas educativas.

Na perspectiva de Ocaña (2009), os atributos que caracterizam os modelos pedagógicos são: i) englobar o conteúdo do ensino, o desenvolvimento do aluno e as características sobre o tema; ii) ter por objetivo a aprendizagem que se concretiza na sala de aula; iii) ser um instrumento de investigação de caráter teórico, desenvolvido para reproduzir idealmente o processo de ensino e aprendizagem; iv) estar suportado por um paradigma para entender, orientar e dirigir a educação. Por sua vez, de forma complementar, Behar (2009) destaca que um modelo pedagógico representa: i) uma estrutura calcada sobre uma determinada concepção epistemológica; ii) uma ou mais teorias educacionais por eixo norteador da aprendizagem; iii) reinterpretações de teorias com base em concepções individuais nem sempre construídas com base em uma determinada teoria. Contudo, os docentes, valendo-se de "um paradigma dominante que, em geral, influencia as teorias de aprendizagem vigentes, assim como outras teorias científicas", embasam-se nestas para construírem seus próprios modelos, decorrentes de suas concepções individuais sobre o processo de aprendizado (BEHAR, 2009, p. 22). Logo, um modelo pedagógico não é sinônimo de teorias de aprendizagem e nem de metodologias de ensino. Diante disso, para a autora, um modelo pedagógico traz consigo uma estrutura alicerçada sobre uma determinada concepção epistemológica e, em consonância, com uma ou mais teorias educacionais a serem utilizadas como eixo norteador da aprendizagem. As concepções epistemológicas dos docentes expressam um paradigma de pensamento educacional sobre a compreensão que eles têm do conhecimento e esta se potencializa nas suas práticas pedagógicas (BECKER, 2001; PINILLA-ROA, 2011).

\subsection{Especificidades da educação a distância}

Inicialmente, o fator primordial da modalidade EAD é a educação. $O$ fato de esta acontecer em local e tempo distinto entre os seus agentes é o que caracteriza essa categoria de ensino e aprendizagem, em nível mundial, exigindo um planejamento detalhado de todo o processo e englobando diferentes aspectos.

Em linhas gerais, a literatura (KEEGAN, 1983; MOORE; KEARSLEY, 2008; BELLONI, 2009; PETERS, 2009; LITTO, 2010) aponta algumas características básicas da EAD: i) representa uma modalidade de educação; ii) apresenta distância física e temporal de seus agentes; iii) executa planejamento prévio das atividades e dos materiais disponibilizados; iv) utiliza processo de ensino e aprendizado intermediado por tecnologias; v) caracteriza-se pela interação entre os agentes mediada por TIC; vi) possibilita maior autonomia dos estudantes; vii) dispõe de professor mediador no processo de ensino e aprendizagem.

Nevado et al. (2001) destaca a interação e a interdependência entre os seus diferentes elementos para o alcance dos objetivos educacionais da EAD, com uma visão integrada e sistêmica. A abordagem sistêmica para adoção dessa modalidade é recomendada por Moore e Kearsley (2008) para garantir a sua eficácia. Os autores entendem haver interdependência entre os diferentes subsistemas da EAD com distintos papéis a desempenhar. Por sua vez, Litto (2010) reforça que a garantia da qualidade e do sucesso das atividades de EAD decorre do planejamento e da preparação prévia por uma equipe de especialistas. Para tanto, necessita de gerenciamento dos recursos e tecnologias, organização, políticas e conteúdos de maneira a poderem interagir e se inter-relacionar no processo de ensino e aprendizagem.

Nesse sentido, de acordo com Bordenave e Pereira (2007, p. 41), os fatores que afetam o processo de ensino podem ser classificados em três: i) aluno: motivações, conhecimentos prévios, relação com o professor, atitude com a disciplina; ii) assunto: estrutura, componentes e relações, tipos de aprendizagem requeridos, ordem de apresentação; iii) professor: situação estimuladora ambiental, comunicação verbal de instruções, informação ao aluno sobre seus progressos, relação com o aluno e atitude com a matéria ensinada.

$\mathrm{Na} \mathrm{EAD}$, esses fatores do processo precisam ser planejados considerando a distância física e temporal entre os agentes envolvidos e o fato de este ser intermediado por alguma forma de TIC. Carvalho, Nevado e Menezes (2007) apontam estruturas de aprendizagens em EAD concebidas pela confluência dos componentes de abordagem pedagógica, internet, inteligência artificial e concepção tempo e espaço, a serem contemplados nas propostas e nos materiais educacionais. Para tanto, um modelo pedagógico para a EAD é compreendido como um sistema "de premissas teóricas que representa, explica e orienta a forma como se aborda o currículo e que se concretiza nas práticas pedagógicas e nas interações professor/aluno/objeto de estudo" (BEHAR, 2009, p. 24).

Da mesma forma, esse modelo impõe que os alunos assumam maiores responsabilidades pelo seu aprendizado, o que implica um planejamento de atividades e de materiais disponibilizados que incentivem a autonomia dos estudantes (PEREIRA et al., 2007; BELLONI, 2009; PETERS, 2009; BEHAR, 2009; LITTO, 2010). Do aluno EAD é esperada organização e disciplina para administrar o tempo de dedicação aos estudos e realização das atividades propostas pelo professor. Ao professor cabe promover a articulação entre os objetivos educacionais e os interesses de aprendizagem dos alunos, tendo por 
papel orientar, auxiliar e criar situações de aprendizagem (PEREIRA et al., 2007; LITTO, 2010).

Para tanto, os professores precisam estar preparados para atuarem nessa modalidade de educação e serem capazes de compreender o processo de ensino e aprendizagem, em consonância com a nova geração de EAD. O que viabiliza outras possibilidades de exploração do conhecimento e de construção coletiva do saber (GOMES, 2013). Sendo assim, a formação de professores necessita contemplar o uso das tecnologias na educação, o que requer "o estudo de propostas teórico-metodológicas e uma ampla divulgação de experiências em EAD, bem como uma reflexão das mesmas" (BEHAR, 2009, p. 17). Prevendo seu planejamento, a autora apresenta os elementos básicos de um modelo pedagógico em EAD: a Arquitetura Pedagógica (AP), representada pelos aspectos organizacionais, de conteúdo, metodológicos e tecnológicos; e a Estratégia para Aplicação (EA), fundamentados por um paradigma epistemológico norteador.

Com base no contexto apresentado, o estabelecimento de um modelo pedagógico aplicado à EAD exige complexidade no seu planejamento decorrente de variantes didáticas específicas, uso contextualizado de tecnologias e uma forma de mediação a ser adotada para promover a educação, entre outros aspectos.

\subsection{Trabalhos relacionados}

Alguns estudos que versam sobre a investigação de modelos pedagógicos para a EAD foram identificados na literatura nacional e internacional (LOAIZA; RAMÍREZ; LINDO, 2001; NEVADO et al., 2001; MARTINS; QUADROS, 2006; PEREIRA et al., 2007; BEHAR, 2009; ANDERSON; DRON, 2011).

O estudo de Loaiza, Ramírez e Lindo (2001) investigou processos metagnitivos com o uso da experiência de mídia virtual, desenvolvido pela Faculdade de Educação da Universidad Tecnológica de Pereira na Colômbia, que visassem a promover a aprendizagem, enfatizando sua aplicação na $\mathrm{EAD}$, o que tem sido chamado de Universidade Virtual. Os autores analisaram a Newport University americana e a Universidad Nacional de Quilmes, da Argentina. Os resultados da pesquisa evidenciaram que a Newport University oferece programas de estudo (graduação, mestrado e doutorado) em EAD com aconselhamento acadêmico individual, para levar o aluno a alcançar a autodisciplina, a autoestima e a realização pessoal, baseado nos aspectos: flexibilidade e estudo independente, e assessoramento individual (tutoria). As atividades de aprendizagem são desenvolvidas por meio de um plano de estudos, contemplando os conteúdos dos programas com a utilização do sistema Syllabus, dividido em várias seções. A avaliação do estudante leva em consideração o cumprimento dos requisitos de cada um dos temas e é submetida à coordenação acadêmica para o orientador realizar a avaliação e fornecer o feedback, permitindo que o estudante faça as correções e encaminhe para a avaliação.

$\mathrm{Na}$ Universidad de Quilmes foi implementado um modelo virtual via internet que fornece ferramentas básicas de navegação, software de gerenciamento e processadores. Os alunos se matriculam numa disciplina obrigatória introdutória, o professor envia a visão geral e o roteiro na primeira semana de aula. A seguir, ocorrem aulas semanais e leituras de textos de orientação. $\mathrm{O}$ aluno torna-se, então, parte da comunidade universitária virtual. E a sala de aula virtual é o espaço de interação entre alunos e professores no Campus Virtual. Esse espaço é composto por diferentes ferramentas que facilitam a comunicação no processo de ensino e aprendizagem: correios ("Avisos do Professor", "Debates" e "Fórum Aberto"), arquivo (literatura obrigatória digitalizada), lista de membros (enviar mensagens e visualizar o currículo) e informações associadas. O programa do curso (disponível impresso e on-line) é uma ferramenta de trabalho que acompanha o processo de aprendizagem do estudante, contribuindo na organização do tempo de estudos. É dividido em unidades temáticas com indicação de materiais didáticos selecionados, organizados de acordo com os problemas do campo disciplinar do plano de trabalho elaborado pelo professor e aprovado pela coordenação do programa. Esse plano é constituído pelos seguintes itens: data (semanal), conteúdos, objetivos, trabalho prático e bibliografias. São fornecidos materiais didáticos impressos (apostilas), especialmente projetados para desenvolver e articular o conteúdo curricular de cada disciplina por pesquisadores, professores e especialistas de diferentes áreas do conhecimento. Estes contêm não só os conteúdos teóricoconceituais, mas também sugerem várias atividades, com base na concepção de aprendizagem como um processo de construção dinâmica e ativa (análise crítica da literatura, estudos de caso e formulação de hipóteses).

Os autores concluem que, embora o modelo de Newport não tenha os benefícios de um campus virtual ou de sistemas de comunicação interativos, apresenta a vantagem de oferecer alta flexibilidade, possibilitando ao estudante poder trabalhar com CDs, material impresso e no tempo por ele escolhido. O processo de formação fortemente personalizado é um dos seus pontos fracos, porque requer habilidades dos alunos para trabalho individual (LOAIZA; RAMÍREZ; LINDO, 2001).

Nevado et al. (2001) estudaram as produções relacionadas à aprendizagem e à internet que orientam a pesquisa e o desenvolvimento entre 500 professores. Os autores investigaram os fatores que afetam o processo de ensino, a partir de Bordenave e Pereira (2007). 
Observaram a carência de conhecimento disponível para aperfeiçoamento dos programas nacionais que visam às transformações na escola e nos cursos de formação de docentes. Os pesquisadores constataram que o conjunto de variáveis que mais impacta o processo de ensino e aprendizagem de acordo com a percepção dos respondentes é: i) motivação (fator aluno), apresentando índice de 0,774 , ii) tipos de aprendizagem requeridos (fator assunto), com índice de 0,741 ; iii) atitude com a matéria ensinada (fator professor), com índice de 0,677. Os resultados revelam que alunos classificam o fator motivacional como o mais importante para seu processo de aprendizagem.

Martins e Quadros (2006) apresentaram uma abordagem integrada para o planejamento de cursos a distância, derivada de investigação pedagógica das diretrizes básicas para integrar as variáveis do processo, tais como currículo, opções de TIC e de gestão. O modelo foi desenvolvido e adotado pela Universidade de Salvador (UNIFACS) para cursos de graduação e pós-graduação em áreas como ciências humanas, engenharia e ciência da computação. A implementação do modelo é focada em decisões pedagógicas orientadas com base em um modelo de currículo flexível, adequado para diferentes cenários de implementação e características dos cursos EAD, ajustáveis às especificidades do público-alvo e às características específicas do curso. O conceito da abordagem pedagógica integrada representou um termo de referência para integrar as diversas dimensões do planejamento do projeto do curso e sua dinâmica de implementação: o modelo de currículo em várias unidades articuladas, a abordagem de gerenciamento de aprendizagem, o sistema de tutoria, a infraestrutura tecnológica, a logística e os métodos e procedimentos de avaliação. As unidades centrais de aprendizagem correspondem às unidades que definem as competências e habilidades a serem desenvolvidas pelas diferentes etapas da formação profissional. São formadas por matrizes temáticas compostas por conjuntos de módulos que definem áreas temáticas curriculares específicas e por matrizes de integração, cujo objetivo é o de integrar, através de experiências de aprendizagem dos alunos, o desenvolvimento do projeto ao longo do curso. Cada módulo integra um conjunto de conteúdos programáticos da área de conhecimento a serem realizados em prazos predefinidos (flows), unidades temporais do curso estabelecidas de acordo com a complexidade do seu conteúdo disciplinar.

Assim, a abordagem pedagógica proposta é composta de unidades curriculares que operam de forma integrada, a fim de promover a articulação entre os conteúdos programáticos, competências e habilidades. As opções de TICs adotadas observaram vários recursos tecnológicos, de acordo com os objetivos do curso, perfil de público e infraestruturas locais disponíveis. Outro aspecto fundamental da abordagem pedagógica integrada é a gestão. O projeto propõe uma nova estrutura de modelo de gestão, composta por coordenação do curso, coordenação técnica e um elemento de coordenação da matriz. Os cursos implementados tiveram seus currículos definidos de acordo com os princípios pedagógicos institucionais básicos gerais, tais como trabalho colaborativo, métodos interdisciplinares, aprendizagem autônoma e aprendizagem significativa.

O estudo de Pereira et al. (2007) teve por objetivo desenvolver um modelo pedagógico de formação de professores para a Universidade Aberta de Portugal. Este foi concebido tendo como base quatro grandes linhas de força: a aprendizagem centrada no estudante, o primado da flexibilidade, o primado da interação e o princípio da inclusão digital (PEREIRA et al., 2007). São elas:

- a aprendizagem centrada no estudante consiste em reconhecer o aluno como produtor de seus conhecimentos e desenvolver situações de ensino que promovam a autonomia, a criatividade, a capacidade de monitorar sua aprendizagem ao longo da vida, a interação entre os estudantes ao invés do foco nos objetivos e materiais educacionais;

- o primado da flexibilidade possibilita ao aluno o aprendizado em qualquer lugar e horário; o modelo prima pela não coincidência no tempo/não coincidência no espaço);

- a interação estudante-estudante compõe-se da criação de grupos de discussão, além da interação aluno-professor; e

- o princípio da inclusão digital consiste na preparação dos estudantes para o uso dos recursos tecnológicos e digitais (literacia digital dos estudantes), levando em conta que a educação on-line exige competências específicas por parte dos estudantes e a possibilidade de inclusão digital (facilitação de acesso) de adultos à EAD sem desenvoltura nas TICs (PEREIRA et al., 2007, p. 10).

Pereira et al. (2007) esclarecem que o modelo pedagógico virtual alicerçado sob essas quatro linhas estruturantes do processo de ensino e aprendizagem está em conformidade com a nova geração da educação on-line. Esta abandonou processos de aprendizagem "essencialmente individuais, autodirigidos e socialmente descontextualizados" (AMANTE, 2011, p. 4). O modelo emergiu da formação em serviço, considerando as competências a desenvolver pelos formandos decorrentes dos perfis traçados pelo Decreto-Lei no 240/2001, da legislação portuguesa, que inclui as dimensões: 
"Profissional, social e ética; desenvolvimento do ensino e da aprendizagem; participação na escola e relação com a comunidade" (AMANTE, 2011, p. 9). Este foi construído para funcionamento em regime on-line, com a utilização da plataforma eletrônica de aprendizagem Moodle e adoção de tutores orientados por docente responsável, permitindo uma interação efetiva, entre formandos e formadores, conferindo à formação uma dimensão coletiva e socialmente contextualizada (PEREIRA et al., 2007; AMANTE, 2011).

Behar (2009) e sua equipe de pesquisadores do Núcleo de Tecnologia Digital Aplicada à Educação (NUTED) investigaram elementos constituintes dos modelos pedagógicos emergentes da EAD. A pesquisa teve por objetivo construir um objeto de aprendizagem a ser utilizado como instrumento norteador da aplicação de modelos pedagógicos na formação de professores para atuarem em cursos nessa modalidade. No estudo, foram identificados os elementos denominados de arquitetura pedagógica (AP) e estratégia de aplicação (EA). A arquitetura pedagógica é constituída de:

(1) fundamentação do planejamento/proposta pedagógica (aspectos organizacionais): onde estão incluídos os propósitos da aprendizagem, organização do tempo e do espaço e expectativas na relação da atuação dos participantes ou da também chamada organização social da classe; (2) conteúdo - materiais instrucionais e/ou recursos informáticos utilizados -, objetos de aprendizagem, software e outras ferramentas de aprendizagem; (3) atividades, interações, procedimentos de avaliação e a organização de todos esses elementos numa sequência didática para a aprendizagem (aspectos metodológicos); (4) definição da plataforma de EAD e suas funcionalidades (aspectos tecnológicos) (BEHAR, 2009, p. 25).

As estratégias para aplicação dão dinamicidade à AP e podem contemplar diferentes estratégias de ação. Representam "o ato didático que aponta a articulação e ajuste de uma arquitetura para uma situação de aprendizagem determinada (turma, curso, aula)" (BEHAR, 2009 , p. 31). Com essa abordagem, foi elaborado um objeto de aprendizagem, denominado de Arquead, disponível no repositório de materiais educacionais do Nuted. Este teve por objetivo abordar diferentes correntes epistemológicas, o uso dos recursos informáticos e as possíveis ações do professor no contexto de EAD.

Anderson e Dron (2011) analisaram três gerações de pedagogia de EAD concentradas nas experiências de aprendizagem decorrentes das concepções de aprendizagem cognitivo-comportamentalista, construtivista social e pedagógico-conectivista. Os pesquisadores concluíram que a EAD de alta qualidade explora todas as três gerações, conforme determinado pelas expectativas de aprendizagem de conteúdo, contexto e aprendizagem.

Mais recentemente, Avendaño-Castro (2013) pesquisou com o objetivo de desenvolver um modelo pedagógico para a educação ambiental de acordo com a urgência da criação de mecanismos e instrumentos que contribuíssem para o desenvolvimento sustentável do planeta.

Em suma, em relação a outros trabalhos da área, o presente estudo contribui na identificação dos aspectos emergentes relativos a concepção epistemológica, contribuições teóricas, configurações organizacionais, metodológicas e tecnológicas para a consolidação dos modelos pedagógicos aplicáveis à EAD.

\section{Procedimentos metodológicos}

O estudo é de natureza aplicada à educação, voltado à formação de docentes de diferentes áreas do saber, com abordagem qualitativa do problema de pesquisa. Quanto aos objetivos, é descritivo, e em relação aos procedimentos técnicos de coleta de dados, é documental (SILVA, 2003; GIL, 2007), pois tem por finalidade conhecer as contribuições científicas que se efetuaram sobre os modelos pedagógicos aplicados à EAD em fontes primárias.

O universo ou população da pesquisa é o conjunto de artigos científicos apresentados no Simpósio Brasileiro de Informática na Educação (SBIE) e publicados nos Anais do Congresso Brasileiro de Informática na Educação (CBIE), considerando a sua relevância e representatividade no Brasil. Magalhães et al. (2013) analisaram 835 trabalhos no SBIE do período de 2001 a 2012 e constataram a participação de 250 diferentes instituições. Dentre estas, universidades públicas e privadas, empresas de desenvolvimento de software, organizações não governamentais e associações, além de órgãos públicos e instituições internacionais dos Estados Unidos, Argentina, Holanda, França e Japão. A EAD e os processos que envolvem o tema desse estudo representaram 19,3\% dos trabalhos (161/835), sendo o segundo tópico mais abordado no evento (MAGALHÃES et al., 2013).

A amostra foi selecionada em abril de 2015, no site do congresso (http://www.br-ie.org/index.php/anaissbie), entre os artigos completos (text full) da área temática de educação do evento, publicados no período de 2001 a 2014, pelas palavras de busca: modelo pedagógico, pedagógico e modelo. E a seleção foi realizada pela leitura dos resumos e, quando necessário, pela leitura completa do material, a fim de relacionar o estudo ao problema desta pesquisa. Os dados do estudo são: i) iden- 
tificativos do trabalho: ano de publicação, título do trabalho, autores; ii) relativos ao modelo nas categorias de análise: paradigma norteador, base teórica, objetivos educacionais, resultados esperados, ação pedagógica proposta, primados da EAD (aprendizagem, flexibilidade, interação e inclusão digital).

Para efetuar a catalogação das visitas ao site, o registro dos dados coletados e a análise qualitativa foi organizado um banco de dados utilizando o Microsoft Office Excel $2007^{\circ}$, a fim de subsidiar a análise realizada. A análise e interpretação dos dados foi realizada com base na fundamentação teórica sobre as especificidades de modelos pedagógicos da EAD nas categorias de análise: paradigma norteador, base teórica, objetivos educacionais, resultados esperados, ação pedagógica proposta, primados da EAD (aprendizagem, flexibilidade, interação e inclusão digital).

\section{ANÁlise de DADOS}

Inicialmente, pelos critérios de busca no SBIE foram listados 198 (cento e noventa e oito) artigos no período de 2001 a 2014. Estes contemplam diferentes abordagens, evidenciadas na Tabela 1.

Tabela 1. Tema principal dos artigos publicados na área de educação no SBIE no período de 2001 a 2014

\begin{tabular}{lcc}
\hline Temática abordada & $\begin{array}{c}\text { Número de } \\
\text { artigos }\end{array}$ & Percentual \\
\hline Objetos de aprendizagem & 40 & 20,2 \\
Ambientes virtuais de aprendizagem & 36 & 18,2 \\
Recursos de acessibilidade & 30 & 15,2 \\
Agentes pedagógicos & 29 & 14,6 \\
Ferramentas de apoio didático & 26 & 13,1 \\
Modelos pedagógicos & 14 & 7,1 \\
Sistemas educacionais & 12 & 6,1 \\
Jogos educacionais & 11 & 5,6 \\
Total & 198 & 100,0 \\
\hline
\end{tabular}

Fonte: Dados da pesquisa (2015).

Dentre os artigos listados (198) pelos critérios de busca, observa-se que a maioria $(81,3 \%)$ diz respeito a estudos relativos a objetos de aprendizagem (20,2\%), ambientes de aprendizagem $(18,2 \%)$, recursos de acessibilidade em AVA (15,2\%), agentes pedagógicos $(14,6 \%)$ e ferramentas de apoio didático $(13,1 \%)$. Esses dados evidenciam que entre os pesquisadores o termo modelo pedagógico tem sido adotado para designar, principalmente, estratégias e ferramentas pedagógicas. Somente 14 (quatorze) artigos (7,1\%) abordaram o tema modelos pedagógicos especificamente, com idealização do processo de ensino e aprendizagem para EAD, apresentando base teórica da aprendizagem norteada por um paradigma epistemológico do processo de ensino e aprendizagem. Esses trabalhos (14 artigos) resultaram na amostra selecionada e analisada a partir das categorias de análise: paradigma norteador, base teórica, objetivos educacionais, resultados esperados, ação pedagógica proposta, primados da EAD (aprendizagem, flexibilidade, interação e inclusão digital). Estas são apresentadas na sequência.

A maioria dos trabalhos analisados (93\%) teve como paradigma norteador uma abordagem cognitivista do conhecimento, com incentivo à participação ativa do estudante no seu processo de aprendizagem. Contudo, um dos trabalhos adotou a abordagem behaviorista da aprendizagem, com foco nos conteúdos e adoção de reforços positivos para respostas corretas. Conforme preconizado por Pereira et al. (2007), a EAD vem evoluindo de concepções instrucionistas para modelos centrados na aprendizagem dos estudantes e no desenvolvimento de competências.

Assim, o embasamento teórico dos modelos analisados, à exceção de um dos trabalhos com abordagem behaviorista, versou sobre as Teorias da Atividade, Aprendizagem Colaborativa, Transformação Cognitiva, Aprendizagem Autorregulada, Construção Social do Conhecimento, Educação Dialógica e Aprendizagem Significativa. Infere-se que estas são decorrentes das concepções individuais dos professores sobre o processo de aprendizado Essas abordagens teóricas, de cunho cognitivistas, demonstram que os docentes têm fundamentado seus modelos pedagógicos em teorias com pressupostos da atividade do sujeito sobre o seu processo de aprendizado. Dessa forma, os fundamentos teóricos para orientação das práticas educativas dos docentes proporcionam maior autonomia aos estudantes, conforme estabelecido na literatura de EAD (KEEGAN, 1983; MOORE; KEARSLEY, 2008; BELLONI, 2009; PETERS, 2009; BEHAR, 2009; LITTO, 2010).

Em relação aos objetivos educacionais propostos e aos resultados esperados, observa-se, na análise dos modelos da amostra, a preocupação dos docentes em oferecer aos estudantes a oportunidade de analisar, debater, planejar, refletir e construir conhecimentos de forma ativa e colaborativa, produzindo comunidades de aprendizagem. Ampliando, dessa forma, a reflexão teórica na articulação da relação dos novos paradigmas educacionais e a formação de uma dimensão coletiva e socialmente contextualizada (PEREIRA et al., 2007; BELLONI, 2009; AMANTE, 2011). As principais proposições estão destacadas no Quadro 1, evidenciando a complexidade dos processos cognitivos e interativos envolvidos na aprendizagem. 
Quadro 1. Relação dos objetivos educacionais propostos e dos resultados esperados nos modelos analisados

\begin{tabular}{|l|l|}
\hline \multicolumn{1}{|c|}{ Objetivos educacionais } & \multicolumn{1}{c|}{ Resultados esperados } \\
\hline Comparar a teoria e a prática $[\ldots]$ & $\begin{array}{l}\text { Apoiar o entendimento dos conceitos básicos e o aprimoramento das } \\
\text { habilidades cognitivas dos estudantes }\end{array}$ \\
\hline Analisar, debater, planejar e construir conhecimentos $[\ldots]$ & $\begin{array}{l}\text { Ampliar a reflexão teórica que articula a relação dos novos paradigmas } \\
\text { educacionais e permitir que os alunos aprendam pelo método da descoberta }\end{array}$ \\
\hline $\begin{array}{l}\text { Discutir e refletir em grupo a partir de atividades de ensino } \\
\text { estruturadas com o propósito de problematização }\end{array}$ & $\begin{array}{l}\text { Organizar procedimentos de investigação, extraindo dados com o propósito } \\
\text { de solucionar problemas propostos em aula }\end{array}$ \\
\hline Realizar experimentos simulados de $[\ldots]$ & $\begin{array}{l}\text { Realizar experimentos com o propósito de solucionar problemas propostos e } \\
\text { promover a reflexão sobre as experiências contínuas de aprendizagem }\end{array}$ \\
\hline Implementar projetos $[\ldots]$ & Construir conhecimento de maneira mais significativa \\
\hline Permitir a construção social do conhecimento & Analisar as atividades construídas a partir da interação \\
\hline Implementar dispositivos móveis & $\begin{array}{l}\text { Praticar a mobilidade tecnológico-educacional; expandir as possibilidades } \\
\text { de navegação introduzidas em narrativas hipertextuais }\end{array}$ \\
\hline
\end{tabular}

Fonte: Dados da pesquisa (2015).

Quanto aos aspectos metodológicos adotados, às ações pedagógicas propostas e às estratégias identificadas nos modelos analisados, de forma geral, $93 \%$ dos modelos pedagógicos da amostra focaram a aprendizagem centrada no estudante ou de forma colaborativa. Somente um dos modelos focou os conteúdos, evidenciando um paradigma instrucionista da aprendizagem. As ações pedagógicas que previram a participação ativa dos estudantes adotaram estratégias que permitem aos estudantes planejar, sequenciar e monitorar a própria aprendizagem, tendo o aluno como foco do processo. Nas ações que adotaram a aprendizagem colaborativa, as estratégias utilizadas são voltadas à resolução de problemas em atividades de grupo.

Em ambas as situações de aprendizagem são adotados procedimentos de comunicação mediados por tecnologias pelos professores ou tutores. A síntese dessa análise está evidenciada no Quadro 2, comparativamente.
Essas correspondências de ações pedagógicas e estratégias estão em consonância com os modelos emergentes da modalidade de EAD, nos quais incentiva-se o desenvolvimento de "situações de ensino que promovam a autonomia, a criatividade, a capacidade de monitorar sua aprendizagem ao longo da vida, a interação entre os estudantes ao invés de ter foco nos objetivos e materiais educacionais" (PEREIRA et al., 2007, p. 10). Observa-se nos modelos analisados que coube ao professor promover a articulação entre os objetivos educacionais e as situações de aprendizagem propostas (PEREIRA et al., 2007; BELLONI, 2009; BEHAR, 2009; LITTO, 2010), de forma a mediar por meio de tecnologias os interesses de aprendizagem dos alunos.

Em relação aos aspectos tecnológicos, todos os modelos pedagógicos analisados ofereceram a flexibilidade temporal e espacial para a realização das atividades propostas, mesmo quando tais modelos eram

Quadro 2. As ações pedagógicas, aprendizagem e estratégias dos modelos analisados

\begin{tabular}{|c|c|c|}
\hline Ação pedagógica & Aprendizagem & Estratégia \\
\hline Flexibilidade cognitiva; prática da autorregulação & \multirow{3}{*}{$\begin{array}{l}\text { Centrada no estudante: participação ativa } \\
\text { e interativa dos alunos }\end{array}$} & Comunidade de aprendizagem \\
\hline $\begin{array}{l}\text { Aulas teóricas e práticas balanceadas; articulação } \\
\text { dialética entre teoria e prática no AVA }\end{array}$ & & Projetos interdisciplinares \\
\hline Diálogo problematizador & & Processo de tutoria pela internet \\
\hline \multirow[t]{2}{*}{ Pesquisa, apresentação, interação e crítica } & \multirow[t]{4}{*}{ Colaborativa } & Utilização de colinks (hipervídeos); \\
\hline & & Padrões pedagógicos \\
\hline Discussões e reflexões do grupo & & Problem Based Learning \\
\hline Perguntas e respostas: webquests & & Trabalhos de grupo \\
\hline Mapa conceitual & Foco nos conteúdos & Resolução de exercícios \\
\hline
\end{tabular}

Fonte: Dados da pesquisa (2015). 
adotados, também, em atividades de ensino presenciais. Assim, os estudantes podiam administrar individualmente o tempo e o local dedicados às atividades, uma vez que eram oferecidos ambientes virtuais de aprendizagem ou via web. Da mesma forma, a interação entre os participantes das atividades de ensino esteve presente em todos os modelos propostos. As principais ferramentas adotadas nas interações foram os fóruns de discussão e chats, para modelos ofertados em AVA, e quadro de avisos e e-mail, para modelos disponibilizados via internet (página web). Contudo, nem todos os modelos apresentados previam atividades de inclusão digital aos seus usuários. Ou, se previam, não foram destacados abertamente nos artigos analisados.

Assim, os princípios estruturais e as funcionalidades dos modelos pedagógicos analisados evidenciaram respeito ao ritmo individual, a formação de comunidades de aprendizagem e de redes de cooperação, conforme preconizado na literatura (BELLONI, 2009; BEHAR, 2009; PETERS, 2009; LITTO, 2010; AMANTE, 2011). Esses resultados revelaram que a maioria dos modelos pedagógicos analisados atendiam aos aspectos emergentes da EAD: serem centrados no aluno, apresentarem princípios da flexibilidade, interação e inclusão digital, conforme destacado por Pereira et al. (2007), previstos nos modelos emergentes da EAD.

\section{Considerações Finais}

De forma abrangente, os modelos pedagógicos da amostra, publicados no SBIE, no período de 2001 a 2014, atendem às características da nova geração de modelos pedagógicos para a EAD. São constituídos de ações pedagógicas e estratégias propostas para desenvolver competências, conforme destacado na literatura. Em linhas gerais, o aluno é agente do seu processo de aprendizagem, o professor é mediador do processo e as trocas entre os colegas são incentivadas.

A formatação metodológica dos modelos pedagógicos analisados revela que os mesmos previam situações de aprendizagem que possibilitassem espaço para (re)construção e difusão do conhecimento. Adotaram ações e estratégias pedagógicas para possibilitar a participação ativa dos alunos nos processos de construção de novos conhecimentos. Dessa forma, corroboram com Peters (2009, p. 155), que assevera que nos espaços virtuais as situações de ensino-aprendizagem "são estruturalmente diferentes daquelas nos espaços reais correspondentes. Preenchê-los com atividades baseadas em modelos de educação tradicional é, portanto, inadequado e, em algumas áreas, levaria à confusão".

Os resultados também demonstraram que o termo modelo pedagógico não está suficientemente consolidado na comunidade científica, gerando uma confusão conceitual a respeito do tema. Esse fato evidencia, também, a necessidade de ampliar as investigações sobre o assunto, a fim de elucidar e difundir o tema no meio científico. Um modelo pedagógico representa um instrumento de investigação de caráter teórico, desenvolvido pelo docente para idealizar o processo de ensino-aprendizagem, sendo suportado por um paradigma epistemológico para entender, orientar e dirigir as práticas pedagógicas. Sendo assim, é desenvolvido para abranger o conteúdo do ensino, promover a aprendizagem e o desenvolvimento do aluno. Deve contemplar uma arquitetura pedagógica (aspectos organizacionais, instrucionais, metodológicos e tecnológicos) e prever as estratégias de aplicação na EAD. Conclui-se que tais aspectos contribuirão para a consolidação do processo de ensino-aprendizagem na EAD.

Nesse sentido, entende-se que há necessidade de formação continuada aos professores, abordando a construção de modelos pedagógicos para a $\mathrm{EAD}$, o que representa um desafio a ser enfrentado pelas instituições de ensino.

Sugerem-se futuras pesquisas relacionadas à abordagem conceitual de modelos pedagógicos e de investigações dos aspectos de inclusão digital e às ferramentas de instrumentalização ao professor com recursos de acessibilidade em AVA.

\section{REFERÊNCIAS}

AMANTE, Lúcia. Formação de professores a distância: a experiência da Universidade Aberta de Portugal. Revista Percursos, Florianópolis, v. 12, n. 01, p. 09-26, jan./jun. 2011.

ANDERSON, Terry; DRON, Jon. Three generations of distance education pedagogy. The International Review of Research in Open and Distributed Learning, v. 12, n. 3, p. 80-97, Mar. 2011.

AVENDAÑO-CASTRO, William Rodrigo. Un modelo pedagógico para la educación ambiental desde la perspectiva de la modificabilidad estructural cognitiva. Revista Luna Azul, Colômbia, n. 36, p. 110-133, jan./jun. 2013.

BECKER, Fernando. Modelos pedagógicos e modelos epistemológicos. In: BECKER, Fernando (orgs.). Educação e construção do conhecimento. Porto Alegre: Artmed, 2001.

BEHAR, Patricia Alejandra. Modelos pedagógicos em educação a distância. In: BEHAR, Patricia Alejandra (orgs.). Modelos pedagógicos em educação a distância. Porto Alegre: Artmed, 2009.

BELLONI, Maria Luiza. Educação a distância. 5.ed. Campinas: Autores Associados, 2009.

BORDENAVE, Juan Díaz; PEREIRA, Adair Martins. Estratégias de ensino-aprendizagem. 14.ed. Petrópolis: Vozes, 2007. 
CARVALHO, Marie Jane Soares; NEVADO Rosane Aragón de; MENEZES, Crediné Silva de. Arquiteturas pedagógicas para educação a distância. In: Aprendizagem em rede na educação a distância: estudos e recursos para formação de professores. Porto Alegre: Ricardo Lenz, 2007.

GALAGOVSKY, Lídia; ADÚRIZ-BRAVO, Agustín. Modelos y analogías en la enseñanza de las Ciencias Naturales: el concepto de modelo didáctico analógico. Enseñanza da las Ciencias, Barcelona, v. 19, n. 2, p. 231-242, 2001.

GIL, Antonio Carlos. Métodos e técnicas de pesquisa social. 5. ed., $8^{\text {a }}$ reimpr. São Paulo: Atlas, 2007.

GOMES, Luiz Fernando. EAD no Brasil: perspectivas e desafios. Avaliação (Campinas) [on-line]. v. 18, n. 1, p. 13-22, 2013.

KEEGAN, Desmond. J. Theoretical principles of distance education. London: Routledge, p. 22-38, 1983.

LITTO, Fredric M. Aprendizagem a distância. São Paulo: Imprensa Oficial, 2010.

LOAIZA, Fernando Romero; RAMIREZ, Hernán Gil; LINDO, Marta Isabel Tobon. La universidad a distancia y la universiad virtual: el modelo pedagógico. Revista de Ciencias Humanas, Colombia, v. 28, p. 143-153, jun. 2001,

MAGALHÃES, Cleyton V. C.; SANTOS, Ronnie E. S.; SILVA, Fabio Q. B. da; GOMES, Alex Sandro Gomes. Caracterizando a pesquisa em informática na educação no Brasil: um mapeamento sistemático das publicações do SBIE. In: Congresso Brasileiro de Informática na Educação. 24, Anais ... CBIE, 2013.

MARTINS, Joberto S. B.; QUADROS, Teresinha. An integrated pedagogical approach for distance learning courses: curriculum, ICT and management. International Federation for Information Processing, v. 210, Education for the 21 Century - Impact of ICT and Digital Resources, eds. D. Kumar, and Turner J. (Boston: Springer), p. 363-367, 2006.

MOORE, Michael G.; KEARSLEY, Greg. Educação a distância: uma visão integrada. Tradução Roberto Galman. 1. ed., $2^{\mathrm{a}}$ reimpr. São Paulo: Cengage Learning, 2008.
MORAN, José Manuel. Aperfeiçoando os modelos de EAD existentes na formação de professores. Educação, Porto Alegre, v. 32, n. 3, p. 286-290, set./dez. 2009.

MOREIRA, José António Marques; BARROS, Rita Manuela; MONTEIRO, Angélica Maria. Autoconceito académico em ambientes virtuais de aprendizagem. Revista Brasileira de Informática na Educação [on-line], v. 22, n. 2, p. 31-46, 2014.

MORENO, Herroro. Modelos educativos y pedagógicos. In: Moreno, H. (org.). Modelos educativos pedagógicos y didácticos. Bogotá: Géminis, 2003. p. 11-95.

NEVADO, Rosane Aragón de; FAGUNDES Léa da Cruz; BASSO, Marcus Vinicius; DUTRA, Ítalo Modesto; PAIM, Marcos Flávio. Um recorte no estado da arte: o que está sendo produzido? O que está faltando segundo nosso sub-paradigma? (premiado). In: Simpósio Brasileiro de Informática na Educação, Vitória, ES. Anais ... SBIE 2001, p. 61-68, nov. 2001.

OCAÑA, Alexander. L. Ortiz. Manual para elaborar el modelo pedagógico de la institución educativa. Colômbia: Editorial Antillas, 2009.

PEREIRA, Alda; MENDES, António Quintas; MORGADO, Lina; AMANTE, Lúcia; BIDARRA, José. Modelo pedagógico virtual da Universidade Aberta: para uma universidade do futuro. Lisboa: Universidade Aberta, 2007.

PETERS, Otto. A educação a distância em transição: tendências e desafios. Tradução de Leila Ferreira de Souza Mendes. São Leopoldo: Editora Unisinos, 2009.

PINILLA-ROA, Análida Elizabeth. Modelos pedagógicos y formación de profesionales en el área de la salud. Acta Médica Colombiana, Colombia, v. 36, n. 4, p. 204-218, out./dez. 2011.

SILVA, Antônio C. R. da. Metodologia da pesquisa aplicada à contabilidade: orientações de estudos, projetos, relatórios, monografias, dissertações, teses. São Paulo: Atlas, 2003.

Submetido em 14/05/2015

Aprovado em 28/03/2016 